\title{
Um Método Geométrico para Construção de Ternos Pitagóricos
}

\author{
João Francisco da Silva Filho(D)
}

\author{
Matheus de Oliveira Bandeira (D)
}

\section{Resumo}

No presente trabalho, apresentamos um interessante método geométrico para a construção de ternos pitagóricos, cujas construções geométricas descritas nos seus passos podem ser reproduzidas usando apenas régua (não graduada) e compasso. Convém salientar que o método supracitado fornece uma interpretação geométrica para as expressões de Euclides e permite-nos obter todos os ternos pitagóricos (primitivos e secundários).

Palavras-chave: Triângulos Pitagóricos; Ternos Pitagóricos; Construções Geométricas.

\section{Abstract}

In this work, we present an interesting geometric method for construction of Pythagorean triples, whose geometric constructions described in its steps can be reproduced by using only straightedge and compass. It should be point out that this method provides a geometric interpretation for the Euclid's formulae and allows us to obtain all Pythagorean triples (primitive and secondary).

Keywords: Pythagorean triangles; Pythagorean triples; Geometric Constructions.

\section{Introdução}

Os triângulos pitagóricos são triângulos retângulos constituídos por lados com medidas inteiras, enquanto ternos pitagóricos são ternos de números inteiros positivos, que correspondem às medidas dos lados de um mesmo triângulo pitagórico. De maneira equivalente, podemos afirmar que ternos pitagóricos são ternos de números inteiros positivos que satisfazem a equação

$$
x^{2}=y^{2}+z^{2},
$$

que é oriunda do Teorema de Pitágoras (cf. [2] e [5]). Dados um terno pitagórico (x,y, z) e um número $\mathrm{p} \in \mathbb{N}$, verifica-se diretamente que (px, py, pz) é um novo terno pitagórico, e, nesse caso, dizemos que os referidos ternos são equivalentes.

Devemos ressaltar que um terno pitagórico é chamado de primitivo, quando suas coordenadas são formadas por inteiros positivos relativamente primos; caso contrário, o terno é chamado de secundário. Na obra Os Elementos, Euclides (ca. 300 a.C.) caracterizou os ternos pitagóricos, 
mostrando que existe uma infinidade deles e apresentando uma forma simples de construí-los. Mais precisamente, Euclides verificou que tomando números $\mathrm{m}, \mathrm{n} \in \mathbb{N}$ com $\mathrm{m}>\mathrm{n}$, tem-se que

$$
\mathrm{x}=\mathrm{m}^{2}+\mathrm{n}^{2}, \quad \mathrm{y}=\mathrm{m}^{2}-\mathrm{n}^{2} \quad \text { e } \quad \mathrm{z}=2 \mathrm{mn}
$$

constituem um terno pitagórico, e tais expressões geram todos os ternos pitagóricos primitivos possíveis (cf. [3] e [4]).

Nesse sentido, desenvolvemos aqui um método geométrico para construir os ternos pitagóricos, estruturado em construções geométricas elementares, tais como: divisão de segmento de reta em partes iguais, construção de reta perpendicular, transportes de segmento de reta e de ângulo etc. Por fim, mostramos que o método descrito está relacionado com as expressões de Euclides, e, a partir dessa relação, concluímos que todos os ternos pitagóricos podem ser obtidos através desse método geométrico.

\section{Resultados Preliminares}

Nesse momento, vamos enunciar e provar os resultados que irão fundamentar o método geométrico a ser apresentado na nossa próxima seção. Diante do exposto, daremos início com uma proposição, que se constitui no resultado chave do trabalho.

Proposição 1. Considere um triângulo retângulo $\mathrm{ABC}$ com ângulo interno $\widehat{\mathrm{A}}$ reto, tal que

$$
\operatorname{med}(\widehat{\mathrm{B}})=2 \theta \quad e \quad \tan \theta=\frac{\mathrm{n}}{\mathrm{m}},
$$

onde $\mathrm{m}, \mathrm{n} \in \mathbb{N}$. Suponha que $\overline{\mathrm{AB}}=\mathrm{p}\left(\mathrm{m}^{2}-\mathrm{n}^{2}\right)$ para algum $\mathrm{p} \in \mathbb{N}$, então,

$$
\overline{\mathrm{AC}}=2 \mathrm{pmn} \quad e \quad \overline{\mathrm{BC}}=\mathrm{p}\left(\mathrm{m}^{2}+\mathrm{n}^{2}\right),
$$

em particular, $\mathrm{ABC}$ é um triângulo pitagórico.

Demonstração. Primeiramente, vamos ilustrar o triângulo ABC, conforme descrito no enunciado, denotando as medidas dos lados BC, AC e AB por a, b e c, respectivamente (cf. Figura 1).

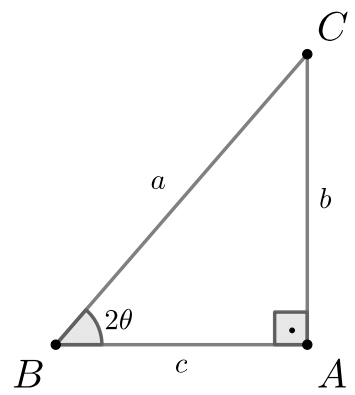

Figura 1: Triângulo retângulo ABC.

Na sequência, vamos traçar a bissetriz BD do ângulo interno $\widehat{B}$ e denotar por d a medida do segmento de reta AD (cf. Figura 2). 


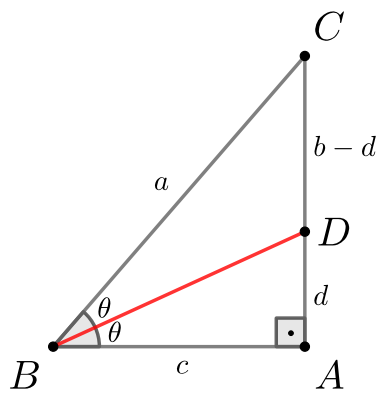

Figura 2: Bissetriz do ângulo $\widehat{B}$.

Observando a Figura 2, aplicamos o Teorema da Bissetriz (cf. Barbosa [2]) e deduzimos que

$$
\frac{\mathrm{b}-\mathrm{d}}{\mathrm{a}}=\frac{\mathrm{d}}{\mathrm{c}}
$$

depois disso, usamos as medidas dos catetos de ABD e a hipótese $\tan \theta=\mathrm{n} / \mathrm{m}$ para obter

$$
\frac{\mathrm{d}}{\mathrm{c}}=\frac{\mathrm{n}}{\mathrm{m}} \text {. }
$$

Decorre das igualdades (1) e (2) que

$$
\mathrm{na}=\mathrm{m}(\mathrm{b}-\mathrm{d})=\mathrm{mb}-\mathrm{nc}
$$

ou, equivalentemente,

$$
\mathrm{mb}=\mathrm{n}(\mathrm{a}+\mathrm{c}) .
$$

Devemos lembrar que o Teorema de Pitágoras estabalece a relação

$$
\mathrm{a}^{2}=\mathrm{b}^{2}+\mathrm{c}^{2}
$$

que junto com a identidade (3) fornece-nos

$$
m^{2} a^{2}=m^{2} b^{2}+m^{2} c^{2}=n^{2}(a+c)^{2}+m^{2} c^{2}
$$

ou,ainda,

$$
\left(m^{2}-n^{2}\right) a^{2}-2 n^{2} a c-\left(m^{2}+n^{2}\right) c^{2}=0 .
$$

Combinando a hipótese $\mathrm{c}=\mathrm{p}\left(\mathrm{m}^{2}-\mathrm{n}^{2}\right)$ com a igualdade $(5)$, chegamos em

$$
a^{2}-2 p n^{2} a-\left(m^{4}-n^{4}\right) p^{2}=0,
$$

que pode ser reescrito na forma

$$
\left(\mathrm{a}-\mathrm{pn}^{2}\right)^{2}=\mathrm{m}^{4} \mathrm{p}^{2} .
$$

Da última igualdade, obtemos a expressão

$$
\mathrm{a}=\mathrm{p}\left(\mathrm{m}^{2}+\mathrm{n}^{2}\right),
$$

então, substituindo-a em (4) junto com $\mathrm{c}=\mathrm{p}\left(\mathrm{m}^{2}-\mathrm{n}^{2}\right)$, vamos ter

$$
\mathrm{b}=2 \mathrm{pmn} \text {; }
$$

daí basta notar que (6) e (7) equivalem a $\overline{\mathrm{BC}}=\mathrm{p}\left(\mathrm{m}^{2}+\mathrm{n}^{2}\right)$ e $\overline{\mathrm{AC}}=2 \mathrm{pmn}$, respectivamente. 
Como aplicação da Proposição 1, obtemos um resultado que nos fornece informações adicionais, no mesmo contexto da proposição citada.

Corolário 1. Considere um triângulo retângulo $\mathrm{ABC}$ com ângulo interno $\widehat{\mathrm{A}}$ reto, tal que

$$
\operatorname{med}(\widehat{\mathrm{B}})=2 \theta \quad e \quad \tan \theta=\frac{\mathrm{n}}{\mathrm{m}},
$$

onde $\mathrm{m}, \mathrm{n} \in \mathbb{N}$. Dessa forma, valem as seguintes afirmações:

a) Se $\overline{\mathrm{AC}}=2 \mathrm{pmn}$ para algum $\mathrm{p} \in \mathbb{N}$, então, $\overline{\mathrm{AB}}=\mathrm{p}\left(\mathrm{m}^{2}-\mathrm{n}^{2}\right)$ e $\overline{\mathrm{BC}}=\mathrm{p}\left(\mathrm{m}^{2}+\mathrm{n}^{2}\right)$.

b) $S e \overline{\mathrm{BC}}=\mathrm{p}\left(\mathrm{m}^{2}+\mathrm{n}^{2}\right)$ para algum $\mathrm{p} \in \mathbb{N}$, então, $\overline{\mathrm{AB}}=\mathrm{p}\left(\mathrm{m}^{2}-\mathrm{n}^{2}\right)$ e $\overline{\mathrm{AC}}=2 \mathrm{pmn}$.

Demonstração. Inicialmente, vamos considerar um triângulo retângulo ABC, conforme enunciado, e em seguida:

- Marcamos um ponto A' sobre a semirreta $\overrightarrow{\mathrm{BA}}$, tal que $\overline{\mathrm{A}^{\prime} \mathrm{B}}=\mathrm{p}\left(\mathrm{m}^{2}-\mathrm{n}^{2}\right)$;

- A partir do ponto A', traçamos um segmento de reta A'C', paralelo ao lado AC e com C' pertencente à semirreta $\overrightarrow{\mathrm{BC}}$ (cf. Figura 3 ).
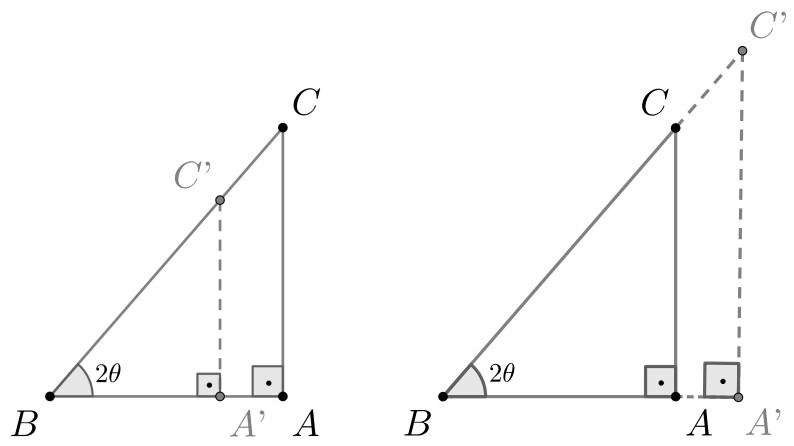

Figura 3: Construção do segmento A'C'.

Observação 1. Na Figura 3, foram ilustrados os dois casos possíveis em que $A^{\prime} \in A B$ e $A^{\prime} \notin A B$, respectivamente.

Podemos afirmar que o triângulo A'BC' (cf. Figura 3) satisfaz às condições da Proposição 1, portanto,

$$
\overline{\mathrm{A}^{\prime} \mathrm{C}^{\prime}}=2 \mathrm{pmn} \quad \text { e } \overline{\mathrm{BC}^{\prime}}=\mathrm{p}\left(\mathrm{m}^{2}+\mathrm{n}^{2}\right),
$$

então, passamos a trabalhar cada item separadamente:

a) Observe que os triângulos retângulos ABC e A'BC' são semelhantes (cf. caso A.A. em [2] e [5]), então, combinando essa informação com a hipótese $\overline{\mathrm{AC}}=2 \mathrm{pmn}$, temos que

$$
\frac{\overline{\mathrm{AB}}}{\overline{\overline{\mathrm{A}^{\prime} \mathrm{B}}}}=\frac{\overline{\mathrm{AC}}}{\overline{\mathrm{A}^{\prime} \mathrm{C}^{\prime}}}=1 \text {, }
$$

logo $\mathrm{AB} \equiv \mathrm{A}$ 'B e $\mathrm{A}=\mathrm{A}$ '. Dessa forma, podemos afirmar que os triângulos ABC e A'BC' coincidem, consequentemente

$$
\overline{\mathrm{AB}}=\overline{\mathrm{A}^{\prime} \mathrm{B}}=\mathrm{p}\left(\mathrm{m}^{2}-\mathrm{n}^{2}\right) \text { e } \overline{\mathrm{BC}}=\overline{\mathrm{BC}^{\prime}}=\mathrm{p}\left(\mathrm{m}^{2}+\mathrm{n}^{2}\right) .
$$


b) De modo análogo, usamos a hipótese $\overline{\mathrm{BC}}=\mathrm{p}\left(\mathrm{m}^{2}+\mathrm{n}^{2}\right)$ junto com a semelhança de $\mathrm{ABC}$ e A'BC' para obter

$$
\frac{\overline{\mathrm{AB}}}{\overline{\mathrm{A}^{\prime} \mathrm{B}}}=\frac{\overline{\mathrm{BC}}}{\overline{\mathrm{BC}^{\prime}}}=1,
$$

daí basta repetir os mesmos argumentos aplicados ao item anterior para concluir a prova.

\section{Construção Geométrica de Ternos Pitagóricos}

Na presente seção, vamos descrever um método geométrico para construir os ternos pitagóricos, que pode ser reproduzido usando apenas régua (não graduada) e compasso. Nessa perspectiva, vamos considerar $n, k \in \mathbb{N}$ na descrição dos passos que compõem o referido método geométrico.

$1^{\circ}$ Passo: Considere um segmento de reta BA.

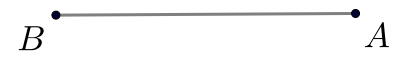

Figura 4: Segmento de reta BA

$2^{\circ}$ Passo: Divida BA em $m=n+k$ partes iguais e adote a unidade de medida $u=\frac{1}{\mathrm{~m}} \overline{\mathrm{BA}}$.

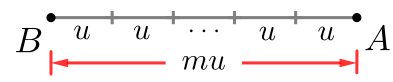

Figura 5: Divisão do segmento BA

Observação 2. Por simplicidade, vamos omitir a unidade de medida u nas demais figuras da seção, ficando subentendido que os comprimentos serão sempre expressos em termos dessa unidade.

$3^{\circ}$ Passo: A partir do ponto A, construa um segmento de reta $\mathrm{AC}$ perpendicular a $\mathrm{BA}$ e com medida $\overline{\mathrm{AC}}=\mathrm{nu}$.

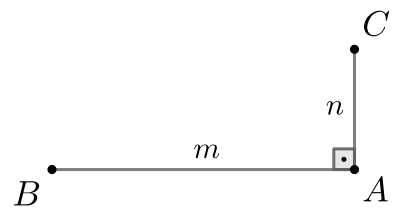

Figura 6: Construção do segmento AC

$4^{\circ}$ Passo: Trace o segmento de reta $\mathrm{BC}$, formando o triângulo retângulo $\mathrm{ABC}$.

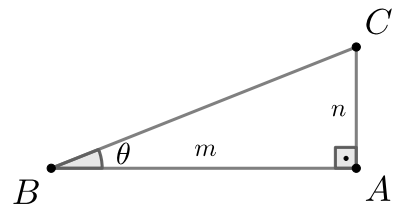

Figura 7: Triângulo retângulo ABC 
$5^{\circ}$ Passo: A partir do ponto B, construa um ângulo $\mathrm{C} \widehat{\mathrm{BD}}$ congruente e adjacente ao ângulo $\mathrm{ABC}$.

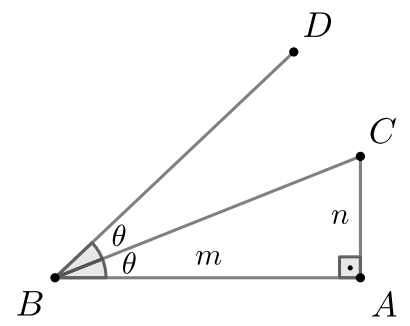

Figura 8: Construção do ângulo C $\widehat{B D}$

$6^{\circ}$ Passo: Sobre a semirreta $\overrightarrow{\mathrm{BA}}$, marque pontos $\mathrm{A}_{1}, \mathrm{~A}_{2}, \ldots, \mathrm{A}_{\mathrm{k}-1}$ e $\mathrm{A}^{\prime}$ distintos entre si e não pertencentes a $\mathrm{BA}$, tais que $\mathrm{AA}_{1} \equiv \mathrm{AC}$ e $\mathrm{BA}_{1} \equiv \mathrm{A}_{1} \mathrm{~A}_{2} \equiv \cdots \equiv \mathrm{A}_{\mathrm{k}-1} \mathrm{~A}^{\prime}$.

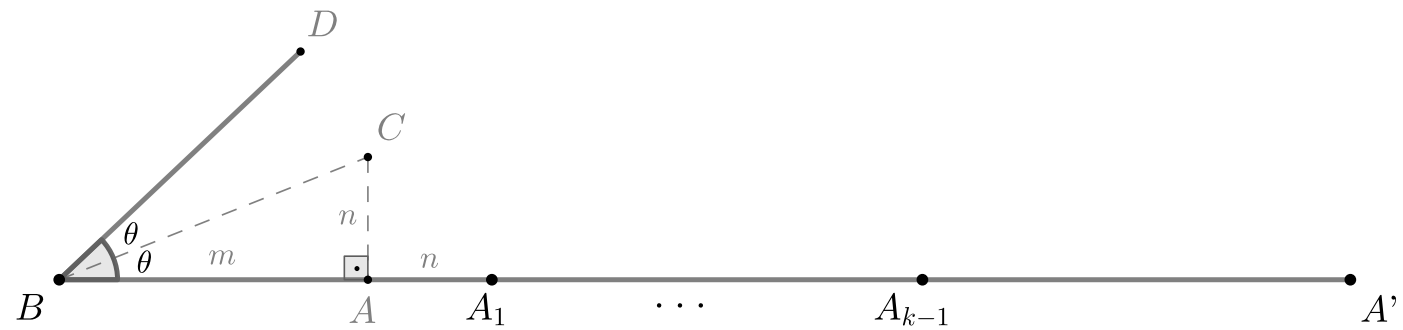

Figura 9: Construção do segmento BA'

Observação 3. Sabendo que os ângulos A $\widehat{B C}$ e ACB, internos ao triângulo ABC (cf. Figura 9), opõem-se a lados com medidas $n$ e $m=n+k$, respectivamente, então, $\mathrm{ABC}$ é menor que $\mathrm{ACB}$. Nessas condições, vale a desigualdade

$$
\operatorname{med}(\mathrm{ABD})<\operatorname{med}(\mathrm{ABC})+\operatorname{med}(\mathrm{ACB})=\frac{\pi}{2},
$$

garantindo assim que A $\widehat{B D}$ é um ângulo agudo.

$7^{\circ}$ Passo: Partindo do ponto A', construa um segmento A'C' perpendicular ao segmento BA', onde $\mathrm{C}^{\prime}$ pertence à semirreta $\overrightarrow{\mathrm{BD}}$ e sua existência decorre da Observação 3 . 


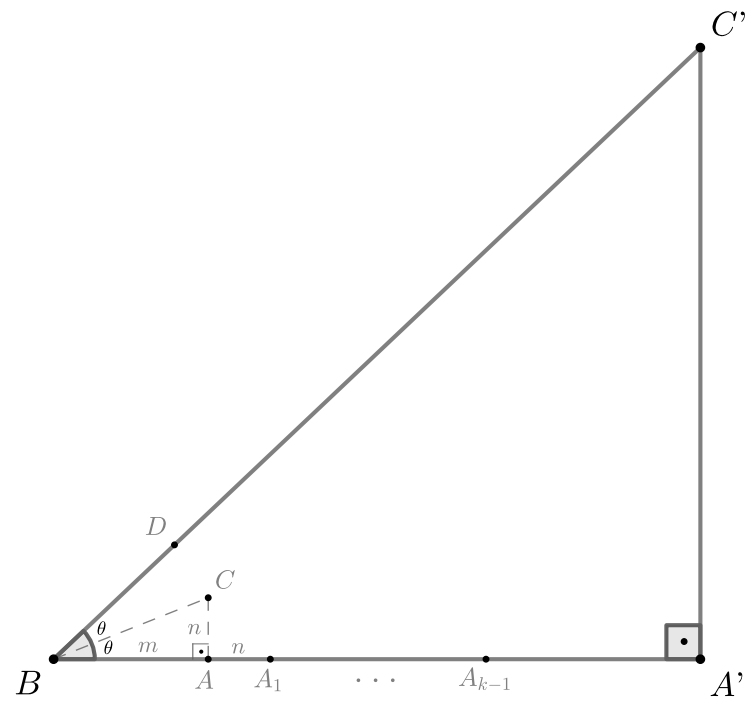

Figura 10: Triângulo retângulo A'BC'

Conclusão: Desde que BA' divide-se em $\mathrm{k}=\mathrm{m}-\mathrm{n}$ segmentos congruentes que medem $(\mathrm{m}+\mathrm{n}) \mathrm{u}$, obtemos

$$
\overline{\mathrm{BA}^{\prime}}=(\mathrm{m}-\mathrm{n})(\mathrm{m}+\mathrm{n}) \mathrm{u}=\left(\mathrm{m}^{2}-\mathrm{n}^{2}\right) \mathrm{u},
$$

implicando pela Proposição 1 que

$$
\overline{\mathrm{A}^{\prime} \mathrm{C}^{\prime}}=(2 \mathrm{mn}) \mathrm{u} \quad \text { e } \quad \overline{\mathrm{BC}^{\prime}}=\left(\mathrm{m}^{2}+\mathrm{n}^{2}\right) \mathrm{u},
$$

portanto, o triângulo A'BC' (cf. Figura 10) é pitagórico, e as medidas dos seus lados formam um terno pitagórico.

Diante da conclusão anterior, observa-se que as medidas dos lados do triângulo A'BC' são dadas pelas expressões de Euclides, e, como tais expressões fornecem todos os ternos pitagóricos primitivos (cf. Hefez [4]), consequentemente todos os ternos pitagóricos primitivos podem ser obtidos a partir do método geométrico apresentado. Sabendo que homotetias podem ser aplicadas a triângulos através de construções geométricas elementares (cf. Seção 5.4 em Wagner [6]), concluímos que os ternos pitagóricos secundários podem ser obtidos aplicando homotetias aos triângulos pitagóricos construídos ao final dos passos descritos pelo nosso método geométrico.

\section{Alguns Exemplos}

Para melhor compreensão do método geométrico apresentado, essa seção apresenta dois exemplos com a construção geométrica de ternos pitagóricos, partindo da bissecção e da trissecção de um segmento de reta.

Exemplo 1. Construindo um terno pitagórico a partir da bissecção de um segmento de reta.

Solução: Conforme os passos do método geométrico descrito na seção anterior, procedemos com a solução do exemplo. 
$1^{\circ}$ Passo: Considere um segmento de reta BA.

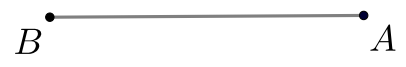

Figura 11: Segmento de reta BA

$2^{\circ}$ Passo: Divida BA em 2 (duas) partes iguais e adote a unidade de medida $u=\frac{1}{2} \overline{\mathrm{BA}}$.

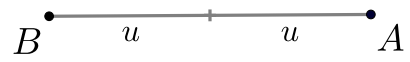

Figura 12: Divisão do segmento BA

$3^{\circ}$ Passo: A partir do ponto A, construa um segmento de reta AC perpendicular a BA e com medida $\overline{\mathrm{AC}}=\mathrm{u}$.

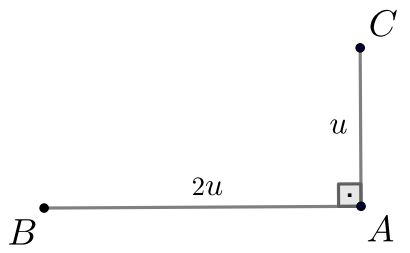

Figura 13: Construção do segmento AC

$4^{\circ}$ Passo: Trace o segmento de reta BC, formando o triângulo retângulo ABC.

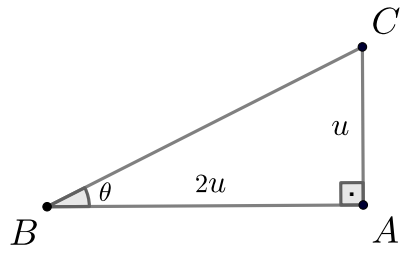

Figura 14: Triângulo retângulo ABC

5' Passo: A partir do ponto $\mathrm{B}$, construa um ângulo $\mathrm{C} \widehat{\mathrm{BD}}$ congruente e adjacente ao ângulo $\mathrm{ABC}$.

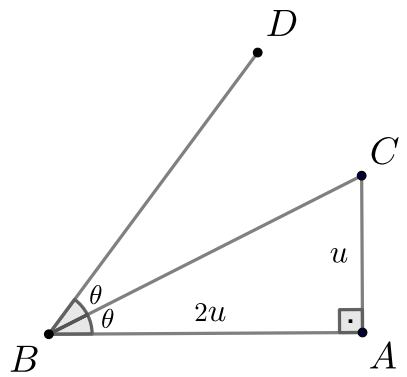

Figura 15: Construção do ângulo C $\widehat{B D}$ 
$6^{\circ}$ Passo: Sobre a semirreta $\overrightarrow{\mathrm{BA}}$, marque um ponto $\mathrm{A}^{\prime}$ não pertencente a $\mathrm{BA}$, tal que $\mathrm{AA}^{\prime} \equiv \mathrm{AC}$.

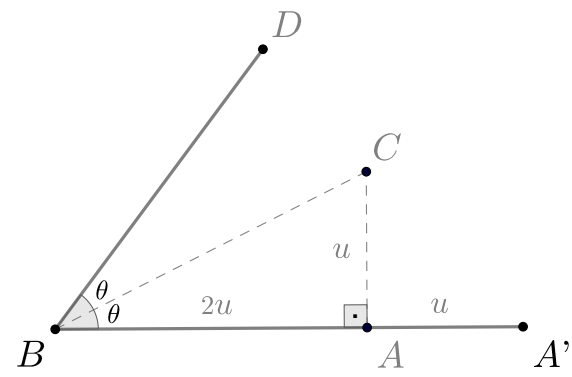

Figura 16: Construção do segmento BA'

$7^{\circ}$ Passo: Partindo do ponto A', construa um segmento A'C' perpendicular ao segmento BA', onde C' pertence à semirreta $\overrightarrow{\mathrm{BD}}$.

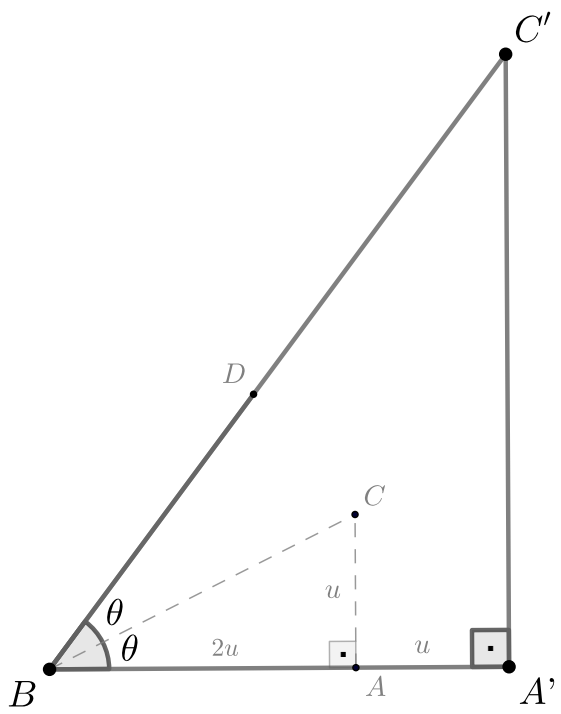

Figura 17: Triângulo retângulo A'BC'

Conclusão: As medidas dos lados do triângulo retângulo A'BC' são dadas por

$$
\overline{\mathrm{BA}^{\prime}}=3 \mathrm{u} \quad\left(\text { por construção), } \overline{\mathrm{A}^{\prime} \mathrm{C}^{\prime}}=4 \mathrm{u} \text { e } \overline{\mathrm{BC}^{\prime}}=5 \mathrm{u} \quad(\text { cf. Proposição } 1),\right.
$$

que correspondem a um terno pitagórico.

Exemplo 2. Construindo ternos pitagóricos a partir da trissecção de um segmento de reta.

Solução 1: De modo análogo ao Exemplo 1, seguimos os mesmos passos executados anteriormente. $1^{\circ}$ Passo: Considere um segmento de reta BA. 


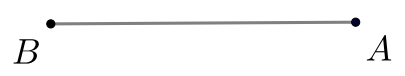

Figura 18: Segmento de reta BA

$2^{\circ}$ Passo: Divida BA em 3 (três) partes iguais e adote a unidade de medida $\mathrm{u}=\frac{1}{3} \overline{\mathrm{BA}}$.

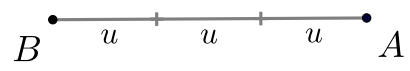

Figura 19: Divisão do segmento BA

$3^{\circ}$ Passo: A partir do ponto A, construa um segmento de reta AC perpendicular a BA e com medida $\overline{\mathrm{AC}}=\mathrm{u}$.

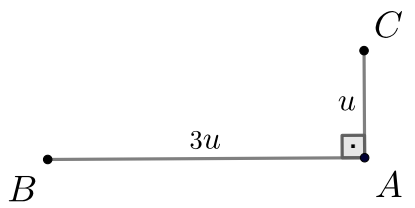

Figura 20: Construção do segmento AC

$4^{\circ}$ Passo: Trace o segmento de reta BC, formando o triângulo retângulo ABC.

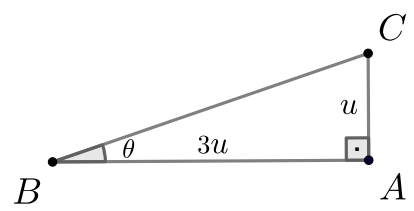

Figura 21: Triângulo retângulo ABC

50 Passo: A partir do ponto $B$, construa um ângulo $C \widehat{B} D$ congruente e adjacente ao ângulo A $\widehat{B} C$.

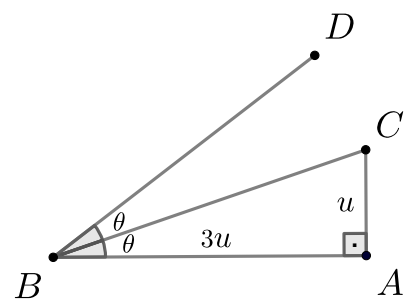

Figura 22: Construção do ângulo C $\widehat{B D}$

$6^{\circ}$ Passo: Sobre a semirreta $\overrightarrow{\mathrm{BA}}$, marque dois pontos $\mathrm{A}_{1}$ e $\mathrm{A}^{\prime}$ distintos e não pertencentes a $\mathrm{BA}$, tais que $\mathrm{AA}_{1} \equiv \mathrm{AC}$ e $\mathrm{BA}_{1} \equiv \mathrm{A}_{1} \mathrm{~A}^{\prime}$. 


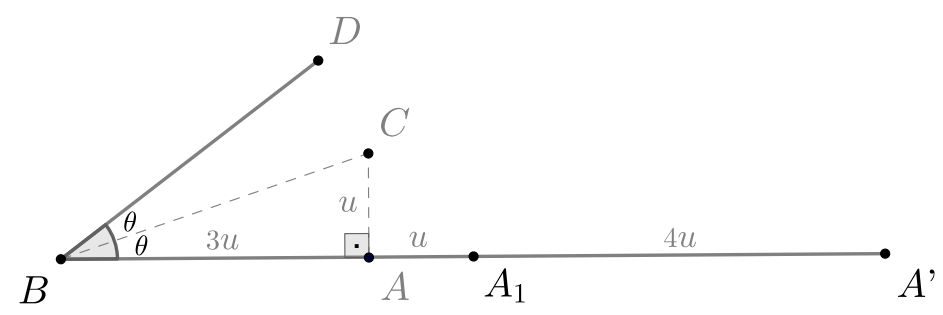

Figura 23: Construção do segmento BA'

$7^{\circ}$ Passo: Partindo do ponto A', construa um segmento A'C' perpendicular ao segmento BA', onde C' pertence à semirreta $\overrightarrow{\mathrm{BD}}$.

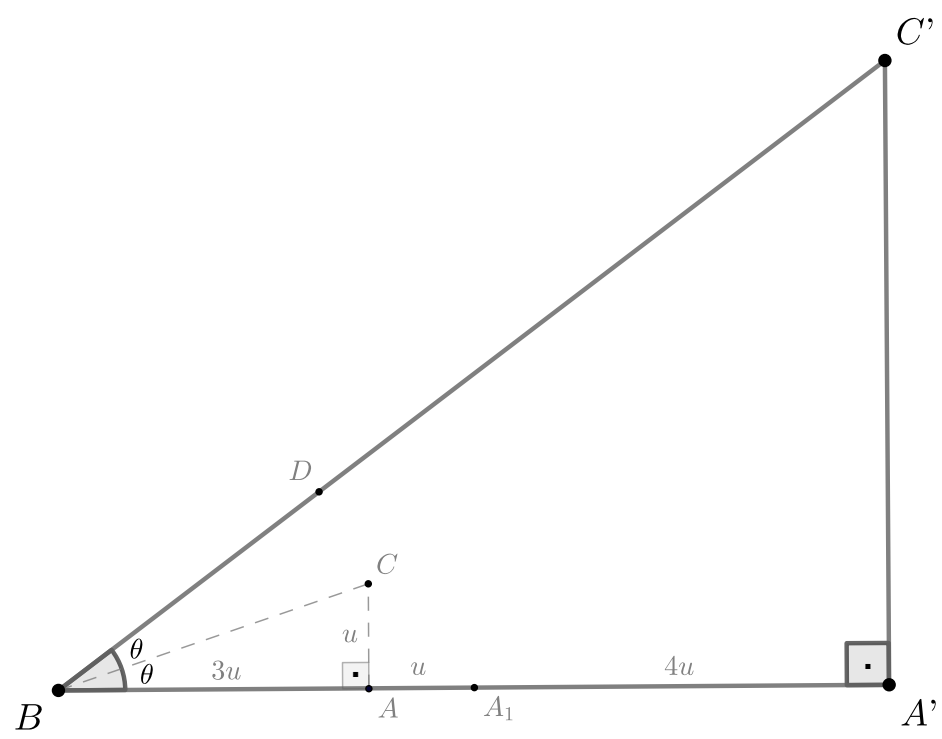

Figura 24: Triângulo retângulo A'BC'

Conclusão 1: As medidas dos lados do triângulo retângulo A'BC' são dadas por

$$
\overline{\mathrm{BA}^{\prime}}=8 \mathrm{u} \text { (por construção), } \overline{\mathrm{A}^{\prime} \mathrm{C}^{\prime}}=6 u \text { e } \overline{\mathrm{BC}^{\prime}}=10 u \text { (cf. Proposição 1), }
$$

que correspondem a um terno pitagórico.

Solução 2: Novamente, vamos repetir os mesmos passos usados nas soluções anteriores.

$1^{\circ}$ Passo: Considere um segmento de reta BA.

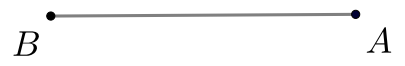

Figura 25: Segmento de reta BA 
$2^{\circ}$ Passo: Divida BA em 3 (três) partes iguais e adote a unidade de medida $\mathrm{u}=\frac{1}{3} \overline{\mathrm{BA}}$.

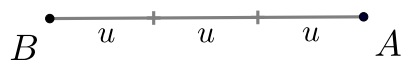

Figura 26: Divisão do segmento BA

$3^{\circ}$ Passo: A partir do ponto A, construa um segmento de reta AC perpendicular a BA e com medida $\overline{\mathrm{AC}}=2 \mathrm{u}$.

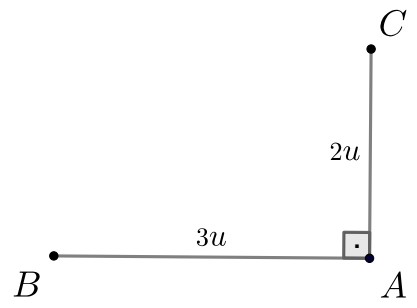

Figura 27: Construção do segmento AC

$4^{\circ}$ Passo: Trace o segmento de reta BC, formando o triângulo retângulo ABC.

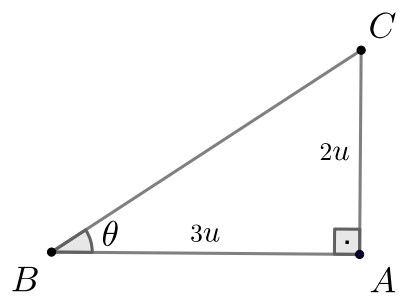

Figura 28: Triângulo retângulo ABC

5' Passo: A partir do ponto $\mathrm{B}$, construa um ângulo $\mathrm{C} \widehat{\mathrm{BD}}$ congruente e adjacente ao ângulo $\mathrm{ABC}$.

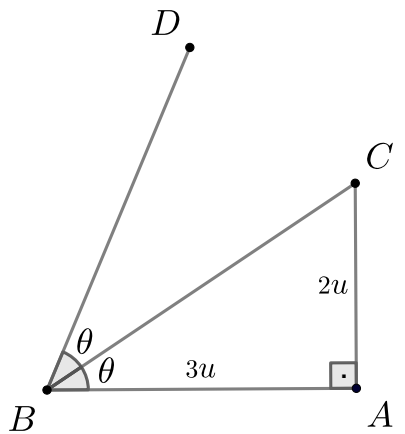

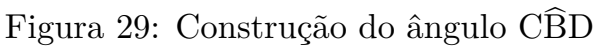


$6^{\circ}$ Passo: Sobre a semirreta $\overrightarrow{\mathrm{BA}}$, marque um ponto $\mathrm{A}^{\prime}$ não pertencente a $\mathrm{BA}$, tal que $\mathrm{AA}^{\prime} \equiv \mathrm{AC}$.

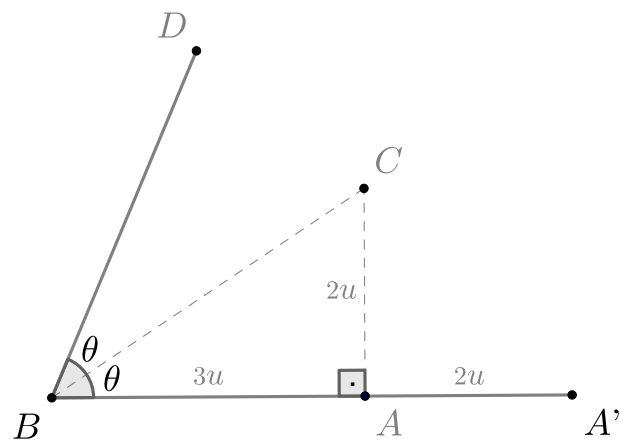

Figura 30: Construção do segmento BA'

$7^{\circ}$ Passo: Partindo do ponto A', construa um segmento A'C' perpendicular ao segmento BA', onde C' pertença à semirreta $\overrightarrow{\mathrm{BD}}$.

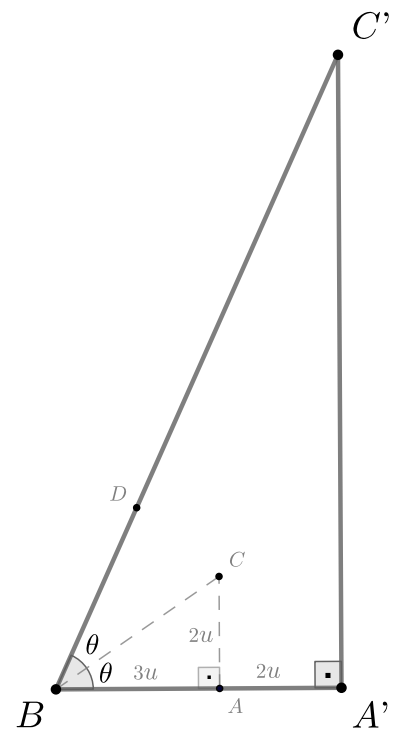

Figura 31: Triângulo retângulo A'BC'

Conclusão 2: As medidas dos lados do triângulo retângulo A'BC' são dadas por $\overline{\mathrm{BA}^{\prime}}=5 \mathrm{u} \quad\left(\right.$ por construção), $\overline{\mathrm{A}^{\prime} \mathrm{C}^{\prime}}=12 \mathrm{u}$ e $\overline{\mathrm{BC}^{\prime}}=13 \mathrm{u}$ (cf. Proposição 1), que correspondem a um terno pitagórico. 


\section{Agradecimentos}

Esse artigo foi parcialmente elaborado durante o desenvolvimento da Monografia de Graduação do segundo autor, que agradece ao CNPq e à Capes pelo apoio financeiro. Por fim, os autores agradecem ao parecerista pelas contribuições e à Revista Professor de Matemática Online pela oportunidade.

\section{Referências}

[1] Bandeira, M. O. Construção Geométrica dos Ternos Pitagóricos. 2020, 32f. Monografia (Graduação) - Unilab, Redenção, 2020.

[2] Barbosa, J. L. M. Geometria Euclidiana Plana. 11ª ed. Rio de Janeiro: SBM, 2012.

[3] Boyer, C. B. História da Matemática. $3^{\text {a }}$ ed. São Paulo: Edgard Blucher, 2012.

[4] Hefez, A. Aritmética. Rio de Janeiro: SBM, 2016 (Coleção Profmat).

[5] Muniz Neto, A. C. Geometria. Rio de Janeiro: SBM, 2013 (Coleção Profmat).

[6] Wagner, E. W. Construções Geométricas. 6 ${ }^{\mathrm{a}}$ ed. Rio de Janeiro: SBM, 2007.

João Francisco da Silva Filho Universidade da Integração Internacional da Lusofonia Afro-Brasileira - Unilab $<$ joaofilho@unilab.edu.br>

Matheus de Oliveira Bandeira Universidade da Integração Internacional da Lusofonia Afro-Brasileira - Unilab <matheusbndr@gmail.com>

Recebido: 17/03/2020

Publicado: 03/06/2020 